\title{
EXPRESSION OF MULTIDRUG RESISTANCE MEMBRANE TRANSPORTER (Pgp) AND p53 PROTEIN IN CANINE MAMMARY TUMOURS
}

\author{
Zsófia KolTAI $^{2^{*}}$ and Péter VAJDOVICH ${ }^{1,2}$ \\ ${ }^{1}$ Department and Clinic of Internal Medicine, Faculty of Veterinary Science, \\ Szent István University, Budapest, Hungary; ${ }^{2}$ Veterinary Haematology and \\ Oncology Centre, Bolgárkertész u. 31, H-1148 Budapest, Hungary
}

(Received 23 May 2013; accepted 31 October 2013)

The aim of this study was to determine the expression rate of P-glycoprotein (Pgp), a multidrug resistance marker and the p53 tumour-suppressor protein in canine mammary tumours. A total of 30 tumours were examined in parallel to patient history. The tumours were allotted to four groups: tubulopapillar carcinomas, complex carcinomas, benign tumours, and other malignant tumours. A monoclonal mouse antibody (C494) was used for the immunohistochemical evaluation of Pgp and a polyclonal rabbit antibody for $\mathrm{p} 53$. We found that the intact ductal epithelium and connective tissue showed pronounced Pgp expression. The most intensive staining was detected in tubulopapillar carcinomas for both Pgp and p53. The expression rate of Pgp and p53 differed significantly between tubulopapillar carcinoma and complex carcinoma, and between tubulopapillar carcinoma and benign mammary tumour, respectively. The expressions of Pgp and p53 highly correlated statistically; therefore, both can determine malignancy in a similar manner. In the case of tubulopapillar carcinomas, more relapsed tumours occurred than in relation to complex carcinomas and other malignant tumours. Pgp expression rate was proportional to the probability of the tumour becoming recidivant postoperatively, as well. These results suggest that routine evaluation of Pgp expression in canine mammary tumours may be prognostically helpful.

Key words: Canine, mammary tumours, multidrug resistance (MDR), Pglycoprotein (Pgp), p53, immunohistochemistry, therapy resistance, prognostic factors

Mammary neoplasm is one of the most common tumour types in female dogs, representing fifty percent of their neoplastic diseases. The most important features of primary tumours are recent rapid growth, size, clinical evidence of invasiveness (fixation to skin or fascia), ulceration, and clinical evidence of inflammatory carcinoma.

*Corresponding author; E-mail: zskoltai@gmail.com; Phone: 0036 (1) 788-0734; Fax: 0036 (1) 788-0734 
Carcinomas frequently metastasise to the inguinal lymph nodes, may enter the pudendal lymph vessels and spread to the internal iliac lymph nodes. In the case of mammary carcinomas the histological subtype has been shown to influence the survival. Anaplastic carcinoma is characterised by shorter postoperative survival (median survival: 2.5 months) than adenocarcinoma (21 months), solid carcinoma (16 months), and other types of tumours ( 24 months). The prognosis of mammary sarcomas is very poor; the median survival time of these patients is usually 9-12 months.

The most common sites of distant metastasis are the lungs, the sublumbar, sternal and prescapular lymph nodes, the liver and, rarely, bone (Sorenmo et al., 2013).

Accurate, precise staging is important before treatment is initiated. The $\mathrm{T}$ (tumour), N (lymph node) and M (metastasis) staging system should be used for dogs with non-inflammatory epithelial tumours and not sarcomas. The most important requirements of staging are to evaluate the primary tumour and the regional lymph nodes, and to attempt to identify any distant metastatic sites, including distant lymph nodes and the lungs (Sorenmo et al., 2013). The magnetic resonance imaging (MRI) technique is available for use in the routine examination of canine mammary glands and staging; the MRI findings are in close correlation with the histological result (Garamvölgyi et al., 2006).

Histological classification is very important for the exact prognosis. Apart from the routine haematoxylin and eosin staining, it is very important to identify the histogenetic origin and the immunophenotype of tumour cells (Sorenmo et al., 2013).

Canine mammary tumours may be characterised by histological classification as malignant or benign tumours. The malignant tumours include non-infiltrating (in situ) carcinoma, complex carcinoma, simple carcinoma (tubulopapillary carcinoma, solid carcinoma, and anaplastic carcinoma), special types of carcinomas (spindle cell carcinoma, squamous cell carcinoma, mucinous carcinoma, and lipid-rich carcinoma), sarcoma, fibrosarcoma, and osteosarcoma, other sarcomas such as carcinosarcoma, carcinoma or sarcoma in benign tumour (Goldschmidt et al., 2011).

In the group of benign tumours, adenoma (simple adenoma, complex adenoma, basaloid adenoma), fibroadenoma (low-cellularity fibroadenoma, highcellularity fibroadenoma), benign mixed tumour, and duct papilloma are known (Hellmén, 1996).

The proliferation capacity of neoplastic cells is one of the most important characteristics of tumours. This determines the degree of malignancy, i.e. the grade. The grade represents important prognostic information, because the behaviour of mammary tumours has been shown to be predicted by the grade. For grading, the degree of differentiation, the mitotic index, the degree of cellular or nuclear polymorphism and the size and number of nucleoli are needed. Some of 
these may be observed by routine histological examination. Other specific tests are the examination of the Ki-67 growth factor (Kiupel et al., 1998) and the silver staining of nucleolar organiser regions (AgNORs) (Juntes et al., 1995; Destexhe et al., 1995; Crocker et al., 1998). There are some other tests as well: malignant tumours have low progesterone expression (Thuróczy et al., 2007), and the evaluation of microvessel density by claudin- 5 immunohistochemistry may give useful additional information on the angiogenic potential of mammary cancers in dogs (Jakab et al., 2008).

Apart from the detection of prognostic markers, the ability of the mammary tumour to develop therapy resistance is equally relevant. Lately, multidrug resistance (MDR) has become one of the most important problems in the management of oncological patients, because in most of the cases MDR is responsible for the failure of chemotherapy. One of the most important factors inducing MDR is the P-glycoprotein (Pgp), an ATP-dependent efflux-pump protein and its classical form is the product of the $m d r-1$ gene. The role of Pgp in the MDR mechanism is that it removes xenobiotics, including chemotherapeutic agents, from the cell; therefore, the level of chemotherapeutic agents cannot reach the minimal intracellular drug concentration necessary for successful chemotherapy. The substrates of Pgp are chemotherapeutic agents such as vincristine, doxorubicin, actinomycin D and mitoxantrone. Approximately $33.3 \%$ of mammary adenomas and $63.2 \%$ of mammary carcinomas express Pgp (Ginn, 1996).

In human and also in veterinary oncology, high Pgp expression is proven to be a negative prognostic factor in many types of tumours. In human oncology, it has been known for a long time that a correlation exists between high Pgp level and MDR in the case of mammary neoplasms (Sanfilippo et al., 1991).

In addition to Pgp, there are other known factors that may also cause MDR, e.g. the multidrug resistance associated protein (MRP), the lung resistance associated protein (LRP), the topoisomerases, and the glutathions (Bergman, 1999).

The $\mathrm{p} 53$ protein is known to be a tumour suppressor. It is an important part of the self-regulating system of the cell. It reduces the expression of the proliferating cell nuclear antigen (PCNA) and the MDR genes, and by this mechanism it blocks tumorigenesis caused by neoplastic mutations. In canine mammary tumours, Veldhoen et al. (1999) have found that the 'germ line' region of the gene encoding the $\mathrm{p} 53$ protein suffered a point mutation, and the protein translated from the gene became inactive. These defective proteins do not have any tumour suppressor effect, but in the cytoplasm or in the nucleus an overexpression can be detected by immunohistochemical examinations, because the hapten serving as the basis of detection is mutated, and the original proteins bind the antibody used in the determination (Lee et al., 2004). The degree of expression of the mutated $\mathrm{p} 53$ shows a correlation with malignancy and shorter survival time (Chu et al., 1998). Mutation of p53 was demonstrated in more than $50 \%$ of human tumours including mammary neoplasms, as well. 


\section{Materials and methods}

\section{Tissue samples}

Mammary tumours of 30 dogs of different ages and breeds were examined. The dogs were operated on at the Department and Clinic of Reproduction, Faculty of Veterinary Science, Szent István University. Age ranged from 5 to 13 years in the following distribution: 5 years old $(n=3), 6$ years old $(n=4), 8$ years old $(n=5), 9$ years old $(n=3), 10$ years old $(n=4), 11$ years old $(n=3)$, 12 years old $(n=6)$, and 13 years old $(n=2)$. Routine histological classification of the tumours resulted in 9 tubulopapillary carcinomas, 12 complex carcinomas, 2 complex adenomas, 2 benign mixed tumours, 1 fibroadenoma, 2 carcinosarcoma, and 1 lipid-rich complex carcinoma.

The samples were fixed in $4 \%$ neutral buffered formalin and embedded in paraffin. For the preparation of the sections we used commercial xylanised slides. The paraffin slides were incubated at $56^{\circ} \mathrm{C}$ for $12 \mathrm{~h}$. Samples were processed up to this phase at the Department of Pathology of the Central Veterinary Institute, Budapest.

\section{The detection of Pgp}

The method was based on the study of Ginn (1996) and on the recommendations of the National Institute of Oncology (Budapest). We performed the immunohistochemistry in the Laboratory of the Department and Clinic of Internal Medicine, Faculty of Veterinary Science, Szent István University, Budapest.

Formalin-fixed sections were deparaffinised and rehydrated through sequential immersions in xylene followed by changes of graded concentrations of ethanol. We blocked the activity of the endogenous peroxidase with $3 \%$ hydrogen peroxide solution. After treatment with appropriate antigen retrieval solution (Retrieve All 1 solution, Signet Laboratories, Dedham, USA, $90{ }^{\circ} \mathrm{C}$, diluted 1:3 with PBS), nonimmune binding of antiserum was blocked by incubating sections in PBS supplemented with 4\% normal horse serum (1:10 dilution, Vector Laboratories, Burlingame, USA). Each tissue section was incubated for $60 \mathrm{~min}$ at room temperature with the primary monoclonal antibody against Pgp (C494, Signet Laboratories) properly diluted in PBS containing 4\% equine serum. Immunohistochemical labelling was performed using the streptavidin-peroxidase procedure. Antigen-bound primary antibody was detected using standard avidinbiotin immunoperoxidase complex (Vectastain Elite ABC Kit). The slides were incubated for $45 \mathrm{~min}$ with biotinylated horse anti-mouse antibody; the chromogen substrate was 3, 3'-diaminobenzidine tetrahydrochloride (DAB, Vector Laboratories). Sections were counterstained with haematoxylin and mounted (DPX mountant, BDH Laboratory Supplies). 
The positive control was a formalin-fixed, paraffin-embedded section of canine liver and kidney tissue from a healthy dog. The negative control was a healthy canine lymph node tissue.

\section{The detection of $p 53$}

The method used for the immunohistochemical detection of p53 was based on the study of Wolf et al. (1997). The staining was performed in the Laboratory of the Department and Clinic of Internal Medicine.

To deparaffinise and rehydrate the samples we used the same method as described previously for the detection of Pgp. Tissue sections were then incubated in preheated $\left(90^{\circ} \mathrm{C}\right)$ citrate solution diluted in PBS $(1: 10)$ as a means of unmasking antigens. Nonimmune binding of antiserum was blocked by incubating sections in PBS supplemented with 4\% normal goat serum (1:10 dilution, Vector Laboratories). The sections were incubated overnight at room temperature with the primary antibody against p53 (1:75 dilution, CM-1, polyclonal rabbit anti-human p53 oncoprotein, Signet Laboratories). The next steps of the staining were the same as those of the Pgp staining, except in this case biotinylated goat anti-rabbit antibody was used. Human breast adenocarcinoma served as positive control, the negative control was healthy canine lymph node.

The distribution and intensity of labelling were analysed by light microscopy. The percentage of Pgp-positive cells was evaluated semiquantitatively and independently by the two authors who were unaware of the origin of samples, by counting the number of labelled cells in 10 fields $(\times 400)$ or at least 500 cells. The expression percentage was determined by dividing the number of labelled cells by the total number of counted cells for each specimen. Nuclear and cytoplasmic expressions were noted but were not included in the scoring.

The expression percentage of p53 cells was determined by methods similar to those described for Pgp. Nuclear and cytoplasmic expressions were not counted separately, because nuclear staining was not seen in $56 \%$ of the specimens. The method used and the results obtained were similar to those of Lee et al. (2004).

\section{Statistical procedures}

Based on the results of histological classification, we divided our patients into 4 groups: tubulopapillary carcinoma $(n=9)$, complex carcinoma $(n=12)$, benign tumour $(n=6)$, other malignant tumour $(n=3)$. Inter-group comparisons were made for the following factors: whether or not the patient had been neutered before the occurrence of mammary tumour; age of the patients at the operation; primary neoplasm or a relapsed tumour; presence of lymph node metastasis or other signs of invasiveness; presence of relapse; occurrence of other types of tumour before/after the operation; whether the cause of death was an oncological disease or some other condition. 

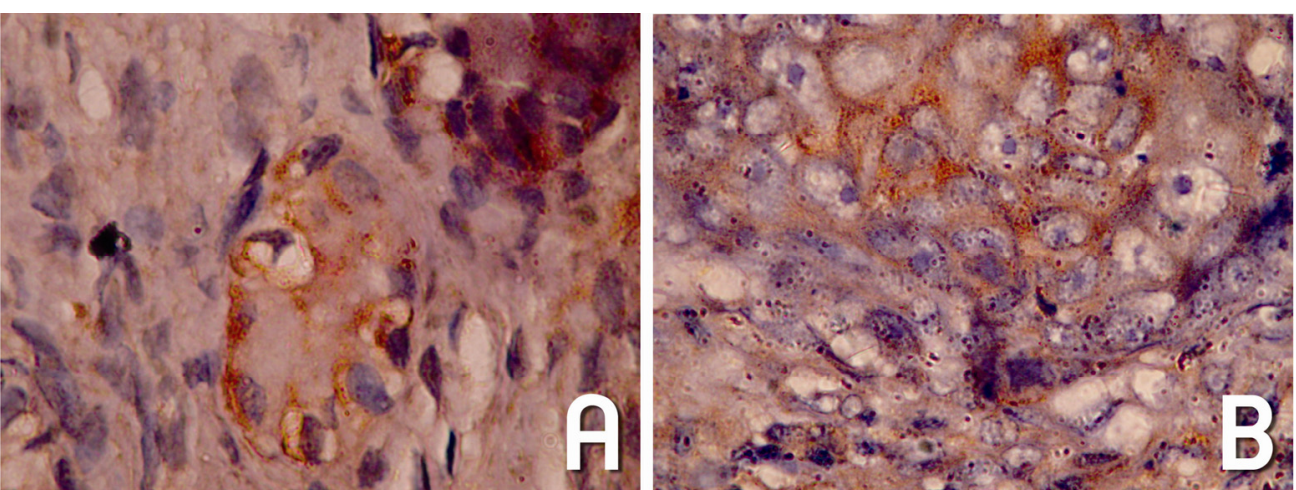

Fig. 1. Expression of Pop and $\mathrm{p} 53$ in canine mammary tumours. The neoplastic cells show a moderate to strong intensity of immunoreactivity. The expression appeared mainly as cytoplasmic staining, with some cells also showing a perinuclear localisation. A: Expression of $\mathrm{p} 53$ in tubulopapillary carcinoma. Avidin-biotin-peroxidase complex method, CM-1 polyclonal antibody, Mayer's haematoxylin counterstain, $\times 1000$ B: Expression of Pgp in complex carcinoma. Avidin-biotin-peroxidase complex method, C494 monoclonal antibody, Mayer's haematoxylin counterstain, $\times 1000$. C: Expression of p53 in complex adenoma. Avidin-biotin-peroxidase complex method, CM-1 polyclonal antibody, Mayer's haematoxylin counterstain, $\times 1000$. D: Expression of Pop in tubulopapillary carcinoma. Avidin-biotin-peroxidase complex method, C494 monoclonal antibody, Mayer's haematoxylin counterstain, $\times 1000$ 
To compare groups, we used Wilcoxon rank-sum test and independent two-sample $t$-test with unequal variance (SAS-STAT software).

Pearson's analysis was also performed in order to find correlations among variables.

\section{Results}

Pgp expression was pronounced in the intact ductal epithelium and in the connective tissue (Fig. 1). The same type of cell occurs in tubulopapillary cancers after neoplastic transformation; therefore, this type of tumour cell shows similarly high Pgp expression. We used Wilcoxon rank-sum test to compare the Pgp and p53 expression rates in the different types of mammary tumours. We observed statistically significant differences in the expression rate of Pgp and p53 between tubulopapillary carcinoma and complex carcinoma, and between tubulopapillary carcinoma and benign mammary tumour (Table 1).

\section{Table 1}

Comparison of different types of tumours in terms of Pgp and p53 expression rate

\begin{tabular}{lcc}
\hline & Pgp (\%) & p53 (\%) \\
\hline I. Tubulopapillary carcinoma & $66.9 \pm 20.1$ & $85.6 \pm 7.3$ \\
II. Complex carcinoma & $35.7 \pm 22.5$ & $66.5 \pm 18.4$ \\
III. Benign tumour & $25.0 \pm 29.5$ & $43.3 \pm 19.3$ \\
IV. Other malignant tumour & $33.3 \pm 29.5$ & $30.0 \pm 15.0$ \\
P-value: I-II & 0.0052 & 0.0146 \\
P-value: I-III & 0.0002 & 0.0847 \\
P-value: I-IV & 0.2431 & 0.1577 \\
P-value: II-III & 0.1683 & 0.2234 \\
P-value: II-IV & 0.9215 & 0.2098 \\
P-value: III-IV & 0.7296 & 0.5665 \\
P-value: I, II, IV-III & 0.001773 & 0.173513 \\
\hline
\end{tabular}

We observed that in patients with tubulopapillary carcinoma, significantly more relapsed tumours occurred later than in patients with any other types of tumours. We found lymph node metastasis associated only with complex carcinomas and carcinosarcomas (Table 2).

The patients' history data were also compared. The fact that the patient had been previously spayed was taken into consideration, because it is well known that neutering decreases the probability of development of canine mammary tumours (Schneider et al., 1969). We compared the age at tumour occurrence, and in these cases we could not find any significant differences among the tumour types. 

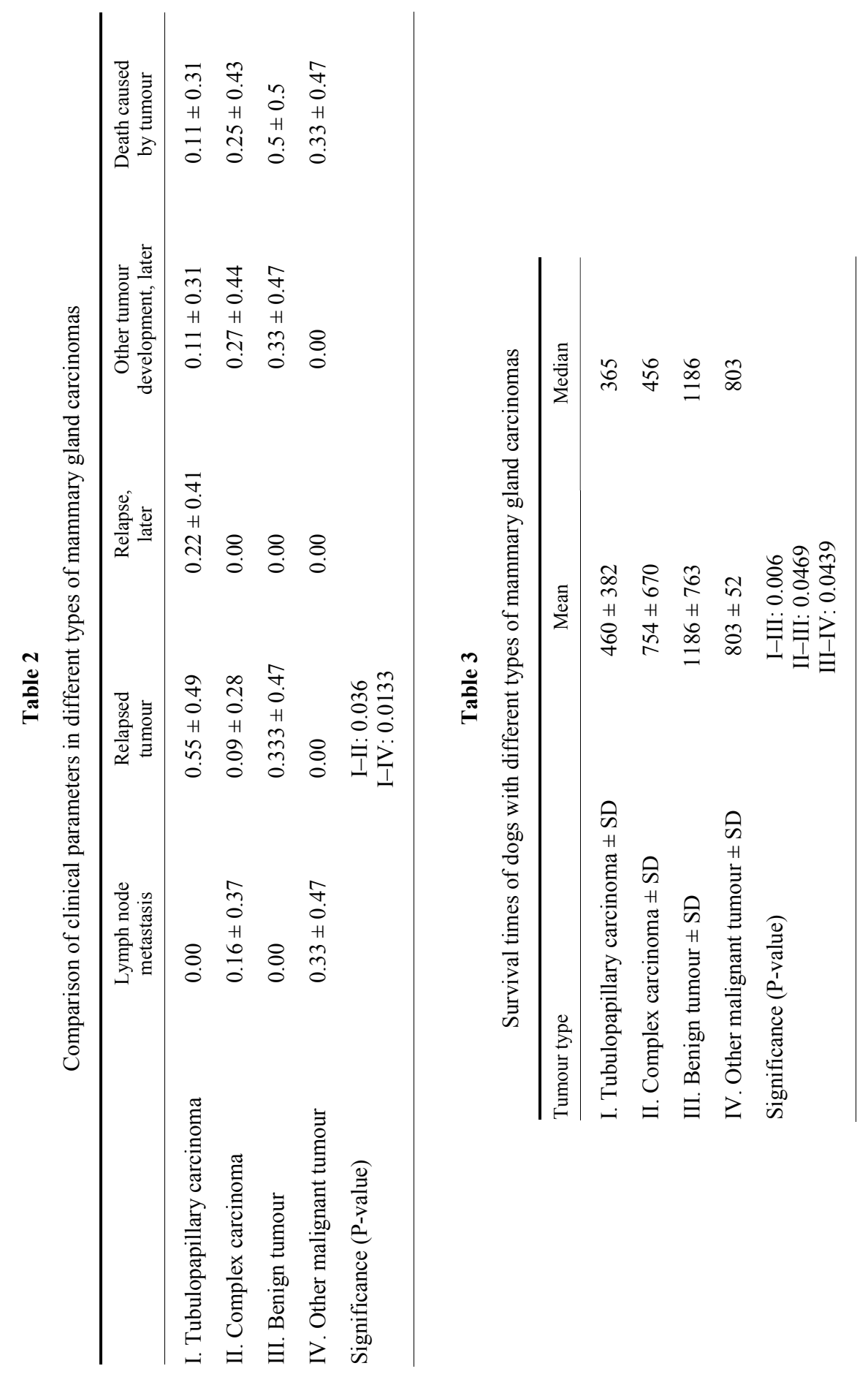
The results indicate that neutering at more than two years of age did not affect the occurrence of the tumours significantly. Dogs with tubulopapillary carcinoma had been spayed in a higher ratio, but at an older age (3-4 years). In the spayed bitches tumours developed by the age of $8-9$ years as well; furthermore, most of these cases later relapsed.

The survival time of dogs with tubulopapillary and complex carcinomas was significantly shorter than that of dogs with benign tumours. Also, dogs with benign tumours had longer survival times than those with other (non-carcinoma) types of tumour (Table 3).

We found that the expressions of Pgp and p53 were correlated. The level of Pgp expression correlated also with the probability of tumour recurrence after the operation and was inversely related to the survival time. An inverse, but nonsignificant, correlation was determined between p53 expression and survival time (Table 4).

Table 4

Correlation of variables in canine mammary gland tumours

\begin{tabular}{lcc}
\hline Parameters compared & Correlation coefficient (R) & \\
\hline Pgp : p53 & 0.55 & $\mathrm{P}<0.01$ \\
Pgp : Relapse, later & 0.484 & $\mathrm{P}<0.01$ \\
Pg: Survival time & -0.287 & $\mathrm{P}<0.05$ \\
p53: Survival time & -0.122 & nonsignificant \\
\hline
\end{tabular}

As regards p53 expression, no correlation was found either between the nuclear and the cytoplasmic expression $(\mathrm{r}=-0.044)$ or between the nuclear expression and the survival time $(\mathrm{r}:-0.145, \mathrm{P}=0.479)$.

\section{Discussion}

In this study we demonstrated that there are significant differences in the expression rate of Pgp between tubulopapillary carcinoma and complex carcinoma as well as between tubulopapillary carcinoma and benign mammary tumour. Because of these high levels, these tumours may be considered the most resistant tumour types. In these tumours, there is a lower chance to perform a successful chemotherapy, especially with drugs that are substrates of Pgp (doxorubicin, vincristine, cyclophosphamide, mitoxantrone).

Petterino et al. (2006) had compared the ratio of Pgp-positive cells in tubulopapillar carcinomas $(49.75 \%)$, complex carcinomas $(40.31 \%)$, benign tumours (31.36\%) and carcinosarcomas (47.28\%). In comparison to their results, the Pgp positivity data obtained in this study were slightly different: $66.94 \%$ in tubulopapillar carcinomas, $35.72 \%$ in complex carcinomas, $25 \%$ in benign tu- 
mours, and $33.33 \%$ in carcinosarcomas. However, the results obtained by the two research groups do not differ significantly.

In contrast to lymphoma, almost all mammary cancer samples showed a certain degree of Pgp expression. Presumably, this phenomenon indicates that if a mammary cancer with lower Pgp expression were treated, after the initial tumour regression a therapy-resistant clone would emerge, which after proliferation would not show regression in response to therapy.

In the present study, Pgp immunostaining values showed a correlation with the survival time regardless of the tumour type. Although the survival time was the shortest in tubulopapillary carcinomas, the Pgp values did not differ significantly from each other.

Regarding the expression of $\mathrm{p} 53$, it is remarkable that we have found positive staining in the benign tumours as well, although at a lower rate than in tubulopapillar or complex carcinomas. The positivity for $\mathrm{p} 53$ is not surprising in adenomas either (Wolf et al., 1997). In another paper it was reported that most carcinomas showed as high as $32 \%$ positive staining for p53 (Rodo and Malicka, 2008). However, in our study a higher expression rate was detected. The source of this discrepancy may be the detection method used, because Rodo and Malicka (2008) counted only stained nuclei, while in the present study we counted both the nuclear and the cytoplasmic staining.

In our study, tubulopapillar carcinomas were tumours originating almost only from recurrence, which was not unexpected as this type of neoplasm often relapses. Concerning age at occurrence, there was no significant difference between the tumour types. In contrast to data reported in the scientific literature (Schneider et al., 1969), neutering of patients at more than two years of age did not provide protection against carcinogenesis. The reason for this difference may be that the dogs examined in this study were probably spayed at an older age.

We demonstrated that the expressions of Pgp and p53 were related. This implies that both the expression of the protein causing therapy resistance and the overexpression of the mutant p53 can help in the detection of malignancy. Therefore, the detection of Pgp expression may be a valuable prognostic parameter in dogs with mammary tumours, even if the animals are not treated with chemotherapy but only by surgery.

\section{Acknowledgements}

We would like to thank Réka Eördögh for her assistance. Financial support from the Hungarian Scientific Research Fund (OTKA), project no. 68376, is acknowledged. 


\section{References}

Bergman, P. J. (1999): Multidrug resistance. In: Bonagura, J. D. and Kersey, R. (eds) Kirk's Current Veterinary Therapy XIII. Small Animal Practice. 13th edition. W. B. Saunders, Philadelphia.

Chu, L. L., Rutteman, G. R., Kong, J. M., Ghahremani, M., Schmeing, M., Misdorp, W., van Garderen, E. and Pelletier, J. (1998): Genomic organization of the canine p53 gene and its mutational status in canine mammary neoplasia. Breast Cancer Res. Treat. 50, 11-25.

Crocker, J., Boldy, D. and Egan, M. (1998): How should we count AgNORs? J. Pathol. 158, 185-188.

Destexhe, E., Vanmanshove, P. and Coignoul, F. (1995): Comparison of argyrophilic nucleolar organizer regions by counting and image analysis in canine mammary tumors. Am. J. Vet. Res. 56, 185-187.

Garamvölgyi, R., Petrási, Zs., Hevesi, Á., Jakab, Cs., Vajda, Zs., Bogner, P. and Repa, I. (2006): Magnetic resonance imaging technique for the examination of canine mammary tumours. Acta Vet. Hung. 54, 143-159.

Ginn, P. E. (1996): Immunohistochemical detection of P-glycoprotein in formalin-fixed and paraffin embedded normal and neoplastic canine tissues. Vet. Pathol. 33, 533-541.

Goldschmidt, M., Pena, L., Rasotto, R. and Zappulli, V. (2011): Classification and grading of canine mammary tumors. Vet. Pathol. 48, 117-131.

Hellmén, E. (1996): The pathogenesis of canine mammary tumours. Cancer J. 9, 282-286.

Jakab, C., Halász, J., Szász, A. M., Kiss, A., Schaff, Z., Rusvai, M., Gálfi, P. and Kulka, J. (2008): Expression of claudin-1, $-2,-3,-4,-5$ and -7 proteins in benign and malignant canine mammary gland epithelial tumours. J. Comp. Pathol. 139, 238-245.

Juntes, P., Cor, A., Pogacnik, M. and Senk, L. (1995): Comparison of the results of the silver nucleolar organizer region (AgNOR) technique on normal and carcinomatous mammary glands of dogs using two different image analysis systems. Zbornik Veterinarske Fakultete Univerza v Ljubljani 32, 7-12.

Kiupel, M., Bostock, D. and Mergmann, V. (1998): The prognostic significance of AGNOR counts and PCNA-positive cell counts in canine malignant lymphomas. J. Comp. Pathol. 119, 407-418.

Lee, C. H., Kim, W. H., Lim, J. H., Kang, M. S., Kim, D. Y. and Kweon, O. K. (2004): Mutation and overexpression of $\mathrm{p} 53$ as a prognostic factor in canine mammary tumors. J. Vet. Sci. 5, 63-69.

Petterino, C., Rosetti, E., Bertoncello, D., Martini, M., Zappulli, V., Bargelloni, L. and Castagnaro, M. (2006): Immunohistochemical detection of P-glycoprotein (Clone C494) in canine mammary gland tumors. J. Vet. Med. 53, 174-178.

Rodo, A. and Malicka, E. (2008): Immunohistochemical expression of protein p53 in neoplasms of the mammary gland in bitches. Pol. J. Vet. Sci. 11, 89-95.

Sanfilippo, O., Ronchi, E., De Marco, C., Di Fronzi, E. and Silvestrini, E. (1991): Expression of Pglycoprotein in breast cancer tissue and in vitro resistance to doxorubicin and vincristine. Eur. J. Cancer 27, 155-158.

Schneider, R., Dorn, C. R. and Taylor, D. O. N. (1969): Factors influencing canine mammary cancer development and postsurgical survival. J. Natl Cancer Inst. 43, 1249-1261.

Sorenmo, K. U., Worley, D. R. and Goldschmidt, M. H. (2013): 27. Tumors of the mammary gland. In: Withrow, S. J. and MacEwen, E. G. (eds) Small Animal Clinical Oncology. Fifth edition. Elsevier Saunders, Missouri. pp. 538-556.

Thuróczy, J., Reisvaag, G. J. K., Perge, E., Tibold, A., Szilágyi, J. and Balogh, L. (2007): Immunohistochemical detection of progesterone and cellular proliferation in canine mammary tumours. J. Comp. Pathol. 137, 122-129.

Veldhoen, N., Watterson, J., Brash, M. and Milner, J. (1999): Identification of tumour-associated and germ line p53 mutations in canine mammary cancer. Br. J. Cancer 81, 409-415.

Wolf, J. C., Ginn, P. E., Homer, B., Fox, L. E. and Kurzman, I. D. (1997): Immunohistochemical detection of $\mathrm{p} 53$ tumor suppressor gene protein in canine epithelial colorectal tumors. Vet. Pathol. 34, 394-404. 